\title{
Mutations in the Drosophila splicing regulator Prp31 as a model for Retinitis pigmentosa 11
}

Malte Lehmann, Sarita Hebbar, Holger Brandl, Weihua Leng, Naharajan Lakshmanaperumal, Sylke Winkler and Elisabeth Knust*

Max-Planck-Institute of Molecular Cell Biology and Genetics, Pfotenhauerstrasse 108

01307-Dresden, Germany

*Corresponding author Tel: +49-351-210-1300

Fax: +49-351-210-1309

e-mail:knust@mpi-cbg.de

\section{Running title}

A Drosophila model for RP11

\section{Keywords}

Retinal degeneration, spliceosome, photoreceptor cells, intragenic complementation, oxidative stress, intron retention 


\begin{abstract}
Retinitis pigmentosa is a clinically heterogeneous disease affecting 1.6 million people worldwide. A growing number of identified disease-causing genes are associated with the spliceosome, but the molecular consequences that link defects in splicing factor genes to the aetiology of the disease remain to be elucidated. In this paper, we present a Drosophila model for Retinitis pigmentosa 11, a human disease caused by mutations in the splicing factor PRPF31. Here, we induced mutations in the Drosophila orthologue Prp31. Mutant flies are viable and show a normal eye phenotype when kept under regular light conditions. However, when exposed to constant light, photoreceptors of mutant flies degenerate, thus resembling the human disease phenotype. Degeneration could be shown to be associated with increased oxidative stress. This increase was in agreement with severe dysregulation of genes involved in oxidation/reduction processes, as revealed by high throughput transcriptome sequencing. In fact, light induced photoreceptor cell degeneration could be attenuated by experimentally reducing oxidative stress. A comparable decrease in retinal degeneration was achieved by raising mutant larvae on a vitamin A-depleted medium, thereby reducing rhodopsin levels. Finally, transcriptome data further uncovered an overall retention of introns in mRNAs. Among those, mRNAs of genes involved in synapse assembly, growth and stability were most prominent. These results point to a multifactorial genesis of light induced degeneration in retinae of Prp31 mutant flies, including transcriptional and splicing dysregulation, oxidative stress and defects in vitamin A metabolism.
\end{abstract}

\title{
Introduction
}

Retinitis pigmentosa (RP; OMIM 268000) is a clinically heterogeneous set of retinal dystrophies, which affects about 1.6 million people worldwide. It often starts with night blindness in early childhood due to the degeneration of rod photoreceptor cells (PRCs), continues with the loss of the peripheral visual field caused by degeneration of cones (tunnel vision), and progresses to complete blindness in later life. RP is a genetically heterogeneous disease, and can be inherited as autosomal dominant (adRP), autosomal recessive (arRP) or $\mathrm{X}$-linked (xIRP) disease. So far $>50$ genes have been identified that are causally related to non-syndromic RP (Daiger et al., 2014) (see RetNet: http://www.sph.uth.tmc.edu/RetNet/disease.htm). Affected genes are functionally diverse. 
Some of them are expressed specifically in PRCs and encode, among others, transcription factors (e. g. $C R X$, an otx-like photoreceptor homeobox gene), components of the lightinduced signalling cascade, including the visual pigment rhodopsin (Rho/RHO in Drosophila/human), or genes controlling vitamin A metabolism (e.g. RLBP-1, encoding Retinaldehyde-binding protein). Other genes are associated with the control of cellular homeostasis, for example CRB1, a gene required for the maintenance of polarity. Interestingly, the second-largest group of genes causing adRP, comprising 7 of 23 genes known, encodes regulators of the splicing machinery. So far, mutations in five PRPF (premRNA processing factor) genes, PRPF3, PRPF4, PRPF6, PRPF8 and PRPF31, have been linked to adRP, namely RP18, RP70, RP60, RP13 and RP11, respectively. PAP1 (Pim1associated protein) and SNRNP2O0 (small nuclear ribonuclearprotein-200), two other genes involved in splicing, have been suggested to be associated with RP9 and RP33, respectively (Maita et al., 2004; Zhao et al., 2009) [reviewed in (Liu and Zack, 2013; Mordes et al., 2006; Poulos et al., 2011; Ruzickova and Stanek, 2016)]. The five PRPF genes encode components regulating the assembly of the U4/U6.U5 tri-snRNP, a major module of the pre-mRNA spliceosome machinery (Will and Luhrmann, 2011). Several hypotheses have been put forward to explain why mutations in ubiquitously expressed components of the general splicing machinery show a dominant phenotype only in PRCs. One hypothesis suggests that PRCs with half the copy number of genes encoding general splicing components cannot cope with the elevated demand of RNA-/protein synthesis required to maintain the exceptionally high metabolic rate of PRCs in comparison to other tissues. Hence, halving their gene dose eventually results in apoptosis. Although this model is currently favoured, other mechanisms, such as impaired splicing of PRC-specific mRNAs or toxic effects caused by accumulation of mutant proteins have been discussed and cannot be excluded to contribute to the disease phenotype [discussed in (Mordes et al., 2006; Scotti and Swanson, 2016; Tanackovic et al., 2011)].

The observation that all adRP-associated genes involved in splicing are highly conserved from yeast to human allows to use model organisms to unravel the genetic and cell biological functions of these genes, which ultimately will provide a mechanistic characterization of the origin of the diseases. In the case of RP11, the disease caused by mutations in PRPF31, three mouse models have been generated by knock-in and knock-out approaches. Unexpectedly, mutant mice did not show any sign of retinal degeneration (Bujakowska et al., 2009). Further analyses revealed that the retinal pigment epithelium, rather than the PRCs, is the primary tissue affected in Prpf31 heterozygous mice (Farkas et al., 2014; Graziotto et al., 2011). 
Morpholino-induced knock-down of zebrafish $\operatorname{Prpf} 31$ results in strong defects in PRC morphogenesis and survival (Linder et al., 2011). Defects obtained by retina-specific expression of zebrafish $\operatorname{Prpf} 31$ constructs that encode proteins with the same mutations as those mapped in RP11 patients (called AD5 and SP117, respectively) were explained to occur by either haplo-insufficiency or by a dominant-negative effect of the mutant protein (Yin et al., 2011). In Drosophila, no mutations in the orthologue Prp31 have been identified so far, but RNAi-mediated knock-down of Prpf31 in the developing Drosophila eye induced, besides strong developmental defects of the eye, signs of PRC degeneration (Ray et al., 2010).

In order to establish a meaningful Drosophila model for RP11-associated retinal degeneration, we isolated two mutant alleles of $\operatorname{Prp} 31, \operatorname{Prp} 31^{P 17}$ and $\operatorname{Prp} 31^{P 18}$, which carry missense mutations that result in exchanges of conserved amino acids. Flies heterozygous for either of these mutations are viable and develop normally. Strikingly, when exposed to constant light, mutant flies undergo retinal degeneration. Degeneration of mutant PRCs was associated with increased oxidative stress. Consistent with this, transcriptome analyses from heads of $\operatorname{Prp} 31^{P 18}$ homozygous flies showed transcriptional dysregulation of genes involved in oxidation/reduction processes. In addition, an overall retention of introns in mRNAs was observed. Retinal degeneration could be ameliorated by supplementing the food with NSC23766, a known inhibitor of NADPH (nicotinamide adenine dinucleotide phosphate)oxidase activity, or by raising larvae on a vitamin A-depleted medium. From these results, we conclude a multifactorial genesis of Prp31-linked retinal degeneration in flies.

\section{Results}

\section{Flies heterozygous for mutations in Prp31 undergo light-dependent retinal degeneration}

It was recently shown that RNAi-mediated knockdown of Drosophila Prp31 in the eye using eyeless (ey)-Gal4 or GMR-Gal4 results in smaller eyes or no eyes at all and degeneration of photoreceptor cells (PRCs) and pigment cells (Ray et al., 2010). Since eyeless is expressed already in the early eye imaginal disc prior to PRC differentiation, some of the defects observed could be secondary, for example as a consequence of defective cell fate specification.

To establish a more meaningful Drosophila model for RP11-associated retinal degeneration, which would allow a deeper insight into the role of this splicing factor in the origin and 
progression of the disease, we set out to isolate specific mutations in Drosophila Prp31 by TILLING (Targeting Induced Local Lesions IN Genomes), following a protocol described recently (Spannl et al., 2017). In total, 2.400 genomes of EMS (ethyl methanesulfonate)mutagenized flies were screened for sequence variants in two different amplicons of Prp31. Four sequence variants were identified, which were predicted to result in potentially deleterious missense mutations. Two of the four lines were recovered from the living fly library and crossed for three generations to wild-type $\left(w^{1118}\right)$ flies to reduce the number of accompanying sequence variants. In two of the lines, named $\operatorname{Prp} 31^{P 17}$ and $\operatorname{Prp} 31^{P 18}$, mutations in $\operatorname{Prp} 31$ could be verified. $\operatorname{Prp} 31^{P 18}$ was viable as homozygotes or in trans over $D f(3 L)$ Exel6262, which removes, among others, the Prp31 locus. In contrast, no homozygous $\operatorname{Prp} 31^{P 17}$ flies were obtained. However, $\operatorname{Prp} 31^{P 17}$ was viable in trans over $\operatorname{Prp} 31^{P 18}$ and over $D f(3 L)$ Exel6262. This suggests that the lethality was due to a second site mutation, which was not removed despite extensive out-crossing. The molecular lesions in the two alleles were mapped in the protein coding region of Prp31. Drosophila PRP31 is a protein of 501 amino acids, which contains a NOSIC domain (named after the central domain of Nop56/SIK1-like protein), a Nop (Nucleolar protein) domain required for RNA binding, a PRP31_C-specific domain and a nuclear localization signal, NLS. $\operatorname{Prp} 31^{P 17}$ contained a point mutation that resulted in a non-conservative glutamine to arginine exchange (G90R) N-terminal to the NOSIC domain. $\operatorname{Prp} 31^{P 18}$ contained a non-conservative exchange of a proline to a leucine residue in the Nop domain (P277L) (Fig. 1A and Supplementary Fig. S1).

Homo- and hemizygous $\operatorname{Prp} 31^{P 18}$, hemizygous $\operatorname{Prp} 31^{P 17}$ flies as well as $\operatorname{Prp} 31^{P 17} / \operatorname{Prp} 31^{P 18}$ transheterozygous flies kept under normal light/dark cycles have eyes of normal size. Histological sections revealed normal numbers of PRCs (distinguished by the number of rhabdomeres) per ommatidium and a normal stereotypic arrangement of PRCs (Fig. 1B-F). This indicates that the development of the retina was not affected by these mutations. However, PRCs of $P r p 31^{P 17} /+, \operatorname{Prp} 31^{P 18} /+$ and $\operatorname{Prp} 31^{P 18} / \operatorname{Prp} 31^{P 18}$ flies showed severe signs of retinal degeneration when exposed to constant light for several days. The same phenotype was observed in PRCs of $\operatorname{Prp} 31^{P 17}$ or $\operatorname{Prp} 31^{P 18}$ hemizygous flies as well as in $D f(3 L)$ Exel6262/+ flies (Fig. 2B-C' and data not shown). After seven days of light exposure, the majority of rhabdomeres of the six outer PRCs, R1-R6, were either completely gone or were strongly reduced in size, and many cell bodies exhibited the typical signs of degeneration, such as condensed chromatin and electron dense material with vesiculation and multivesicular bodies (Fig. 2B-C'; quantification in Fig. 2G). No significant differences were observed between the two alleles. R7 is preserved in most ommatidia. Strikingly, PRCs of 
transheterozygous $\operatorname{Prp} 31^{P 17} / \operatorname{Prp} 31^{P 18}$ flies showed less degeneration in comparison to those of flies heterozygous for either of these alleles (Fig. 2E, E'). Many intact rhabdomeres were present, although some of them showed first signs of degeneration. Only few PRCs showed the dark staining typical for degenerating cells (quantification in Fig. 2F). In contrast to other fly models of retinal degeneration, for example $c r b$ (Pocha et al., 2011), Prp31 mutant flies aged for 30 days under regular light/dark conditions did not show major signs of degeneration (data not shown). To summarise, the isolated mutant Prp31 alleles reveal dominant, lightdependent retinal degeneration, similar as mutations in RP11 patients, and show intragenic complementation.

\section{$\operatorname{Prp31}^{P 18}$ mutant flies exhibit increased oxidative stress signalling}

Photoreceptors have an extraordinary oxygen consumption due to their high biosynthetic activity, which is required to continuously replenish the photosensitive apical membrane (Ng et al., 2015; Yu and Cringle, 2001). In addition, although PRCs are specialised for light reception to initiate phototransduction, light at the same time is a stress factor and induces increased production of reactive oxygen species (ROS) (German et al., 2015). Increased levels of cellular ROS, in turn, induce antioxidant responses, which include the expression of proteins against oxidative stress, e.g. superoxide dismutase (SOD) or glutathione Stransferase. Their activity can prevent the cell from the detrimental consequences of oxidative stress, such as increased lipid oxidation or damage of proteins and DNA (Tomanek, 2015). In photoreceptor cells, a failure of the antioxidant machinery to neutralise increased levels of ROS can lead to light-dependent retinal degeneration, for example in fly PRCs mutant for $c r b$ (Chartier et al., 2012).

This raised the question whether flies mutant for $\operatorname{Prp} 31$ are subject to increased oxidative stress. To address this question, we analysed $\operatorname{Prp} 31^{P 18}$ mutant flies that carried the GstD-GFP reporter transgene. This reporter expresses GFP under the control of upstream regulatory sequences of glutathione S-transferase ( $g s t D 1$ ), one of the genes involved in detoxification, whose expression is activated by oxidative stress (Sykiotis and Bohmann, 2008). The expression of this reporter has been shown to correlate with ROS levels, as revealed by the ROS-sensitive dye Hydro-Cy3 in the midgut of adult flies stressed by feeding bacteria (Jones et al., 2013). GFP expression was determined by measuring fluorescence levels in lysates of flies kept for two days on standard food and of flies kept for three days on food containing $5 \%$ 
hydrogen peroxide $\left(\mathrm{H}_{2} \mathrm{O}_{2}\right)$, an established oxidative stressor. Compared to control flies, $\operatorname{Prp} 31^{P 18}$ heterozygous flies kept on normal food showed a $25 \%$ increase in GstD-GFP expression (Fig. 3A). This suggests that $\operatorname{Prp}^{P 18}$ mutants are under increased levels of oxidative stress signalling already under standard/basal conditions. Upon exposure to $5 \%$ $\mathrm{H}_{2} \mathrm{O}_{2}$, lysates from control flies displayed an $18 \%$ increase in GstD-GFP expression, while lysates from $\operatorname{Prp}^{P 1}{ }^{P 18}$ mutant flies exhibited a $47 \%$ higher level of GFP compared to control flies kept on standard food (Fig. 3A). To corroborate these findings, GFP expression was examined in-situ by immunostaining of adult eye tissue. In control eyes, GstD-GFP expression was high in the pigment and cone cells. Interestingly, no GstD-GFP expression was detected in the photoreceptor cells themselves (Fig. 3B-B'). In eyes of $\operatorname{Prp} 31^{P 18} /+$ flies GstD-GFP levels were strongly increased in cone and pigment cells (Fig. 3C-C'). Taken

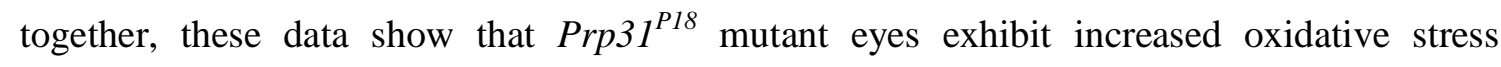
signalling already under normal conditions, particularly in cone and pigment cells.

\section{Prevention of light-induced degeneration of Prpf31 mutant photoreceptor cells}

It is well documented that increased ROS levels in both vertebrate and invertebrate PRCs can lead to neuronal degeneration upon exposure to additional stress, such as light stress (Punzo et al., 2012). Therefore, we assumed that the observed increase in the antioxidant response in the retina of Prp31 mutant flies is the result of increased ROS levels, and that these could be the cause for light-dependent retinal degeneration. To test this assumption, we experimentally blocked one of the major sources of cellular ROS production, mediated by the NOX (NADPH oxidase) family (Bedard and Krause, 2007). One subunit of NOX is Rac1, a member of the small GTPase protein family, which is involved, besides cytoskeletal remodelling, in the generation of ROS (Hordijk, 2006). Previous reports suggested that light can stimulate Rac1 (Balasubramanian and Slepak, 2003; Belmonte et al., 2006), which in turn results in enhanced NADPH-oxidase activity and thus increased ROS levels. In mice, constitutively active Rac1 can promote photoreceptor degeneration (Song et al., 2016), while depletion of Rac1 in photoreceptor cells was able to protect the cells from photooxidative stress (Haruta et al., 2009; Song et al., 2016). Furthermore, inhibiting Rac1 activity in a fly model for RP12, caused by loss of $c r b$ function, reduced ROS production and prevented light-dependent retinal degeneration (Chartier et al., 2012). To test whether NOX is involved in light-dependent retinal degeneration in $\operatorname{Prp} 31^{P 18}$ homozygous mutants, we blocked Rac1 activation by feeding flies for two days before and during exposure to light with NSC23766, a selective inhibitor of 
the Rac1-GEF interaction and hence of Rac1 activation (Nassar et al., 2006). After lightexposure, treated animals exhibited a strongly reduced number of apoptotic PRCs (Fig. 3D). From these results we conclude, that increased NADPH-oxidase activity, which is likely followed by increased accumulation of ROS, is a major trigger of light-dependent PRC degeneration in $\operatorname{Prp} 31^{P 18}$ homozygous mutant flies.

It has been suggested that retinal degeneration in $\operatorname{Prpf} 31$ mutant mice is caused by accumulation of truncated, and hence toxic rhodopsin proteins, which is generated as a result of improper splicing of the rhodopsin pre-mRNA (Yuan et al., 2005). To find out whether rhodopsin accumulation may contribute to light-dependent degeneration in Prp31 mutant flies, we raised larvae in a food lacking vitamin A, the precursor for retinal, the cofactor covalently bound to opsin. This treatment was shown to prevent light-dependent retinal degeneration in crb mutant PRCs (Johnson et al., 2002). As shown in Fig. 4, lack of dietary vitamin A strongly suppressed light-dependent degeneration of PRCs in Prp31 mutant flies.

\section{Prp31 mutants reveal mis-regulation of genes involved in oxidation-reduction}

In mouse and zebrafish, PRC degeneration due to loss of PRPF31 has been associated with a general reduction in biosynthetic activity as well as with impaired splicing of PRC-specific mRNAs, such as rhodopsin, RDS (Peripherin) or Fascin (FSCN2) mRNA (Linder et al., 2011; Mordes et al., 2006; Tanackovic et al., 2011; Yin et al., 2011; Yuan et al., 2005). To get a deeper insight into the mechanisms by which Drosophila Prp31 prevents retinal degeneration, we performed whole transcriptome analysis from RNA isolated from heads of

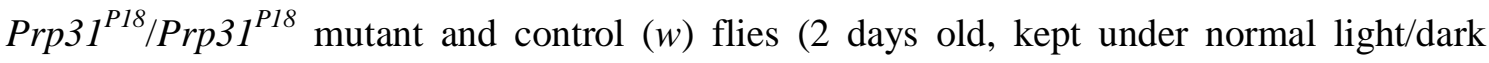
conditions). For each genotype three biological replicates were analysed. Overall, 115 genes were significantly (q-value cut-off 0.01 ) and differentially expressed (2-fold change) in $\operatorname{Prp} 31^{P 18}$ homozygous fly heads compared to $w$ control flies. Of these, 53 were up-regulated and 62 were down-regulated (Suppl. Table S1, S2). Differentially expressed genes were categorized using the Gene Ontology (GO)/PANTHER classification system (Mi et al., 2013; Thomas et al., 2003), based on the predicted protein class of their gene products. Within the groups of up- and down-regulated genes, the three most prominent categories comprised genes with hydrolase, oxidoreductase, and transporter activities (Fig. 5A, B).

We then focused on the 53 genes with increased expression in the mutants (Table S1). The largest group of those that match a GO term $(n=9)$ fall into the GO term: oxidoreductase (Fig. 
5A). This group includes genes of the Cytochrome-P450 family as well as the gene cinnabar $(c n)$, which encodes a monoxygenase with a predicted $\mathrm{NAD}(\mathrm{P}) \mathrm{H}$ oxidase activity, and plays a role in the pigment biosynthetic pathway in the eye. We also observed an up-regulation of $w$, encoding a transporter, and $R h 6$, which encodes an opsin expressed in a subset of photoreceptors. Amongst the 62 genes with decreased expression in the mutant (Table S2) the three most abundant classes matching a GO term comprised genes encoding hydrolases, oxidoreductases, and transporters (Fig. 5B). The group of hydrolases contains a chitinase (Cht3). Relevant for eye function are genes encoding transporters, including the gene scarlet $(s t)$, which is known to be involved in pigment formation in the retina.

Since we observed overlapping categories in genes with increased and decreased expression in the mutants, we evaluated all 115 differentially expressed genes by applying statistical enrichment tests. Through this we identified enrichment for oxidoreductase activity, heme binding, tetrapyrrole binding, and lyase activity (Fig. 5C), corroborating our qualitative results. It should be noted that with the exception of Desat 2 in the first group (oxido-reductase activity) the first four classes comprise the same 8 members of the Cytochrome-P450 (Cyp) gene family.

\section{Prp31 mutants exhibit increased intron retention}

Since Prp31 encodes a splicing factor, we asked whether a mutation in this gene impairs splicing on a genome-wide level, as has been described in zebrafish eyes with reduced Prpf31 activity (Linder et al., 2011), in Drosophila embryos mutant for Prp19 (Sauerwald et al., 2017), and in lymphoblasts derived from patients mutant for PRPF3, PRPF8 or PRPF31(Tanackovic et al., 2011). Our data reveal that from a total of 49345 introns encoded in the genome, present in 13917 genes, 131 were significantly retained (FDR<0.1) in the mutant transcriptome, representing 127 genes (Table S3). To further evaluate the genes showing intron retention, we applied statistical enrichment tests. Strikingly, introns derived from genes falling into the categories of synaptic vesicle cycle/localization/exocytosis/transmission were particularly retained in the mutant (Fig. 6).

Taken together, we established a fly model for RP11, a retinal disease caused by mutations in the highly conserved splicing factor PRPF31. We show that mutations in Drosophila Prp31 induce light-dependent retinal degeneration, thus mimicking the symptoms of the human disease. We further uncovered major dysregulation of the transcriptome of mutant fly heads. 
The nature of the mis-regulated genes as well as the result of rescuing the mutant phenotype let us to conclude that light-dependent PRC degeneration of Prp31 mutant eyes is of multifactorial origin, including increased oxidative stress, defects in rhodopsin metabolism, and splicing defects in genes involved synaptic transmission.

\section{Discussion}

Here we present a fly model for RP11, an autosomal-dominant human disease leading to blindness, which is caused by mutations in the splicing regulator PRPF31. Our results reveal that, similar as in humans, mutations in the Drosophila orthologue Prp31 lead to PRC degeneration under light stress, thus mimicking major features of RP11-associated symptoms. Similar as in human, mutations in Drosophila Prp31 lead to retinal degeneration when heterozygous. This is in stark contrast to a mouse heterozygous for Prpf31, which did not show any signs of retinal degeneration (Bujakowska et al., 2009). Different studies showed late-onset defects in the retinal pigment epithelium of Prpf31 mutant mice (Farkas et al., 2014; Graziotto et al., 2011). Most of the mutations in human PRPF31 linked with RP11 are associated with reduced PRPF31 mRNA, suggesting that these are loss-of-function alleles (Rio Frio et al., 2008; Ruzickova and Stanek, 2016).

Data presented here let us to conclude that the two missense mutations mapped in $\operatorname{Prp} 31^{P 17}$ and $\operatorname{Prp} 31^{P 18}$ represent hypomorphic conditions, which reduce, but do not abolish the function of the protein. First, unlike in humans, the two Drosophila alleles characterized here are hemizygous and homozygous (in the case of $\operatorname{Prp} 31^{P 18}$ ) viable and fertile. Second, $\operatorname{Prp} 31^{P 17} / \operatorname{Prp}^{P 1} 1^{P 18}$ flies showed intragenic complementation and exhibited only a mild degenerative phenotype. Intragenic (interallelic) complementation is a rare event, and is often explained by the fact that two or more defective proteins can form functional multimers, if their mutations reside in different domains. This has been shown, for example, for mutations affecting Drosophila Dynein (Gepner et al., 1996) or Posterior sex combs (Psc), a member of the homeotic Polycomb Group (PcG) proteins involved in epigenetic silencing (Wu and Howe, 1995). In fact, the mutations in the two established Prp31 fly lines reside in different parts of the protein, namely N-terminal to the NOSIC domain in $\operatorname{Prp}^{2} 1^{P 17}$ (G90R) and in the Nop domain in $\operatorname{Prp}^{P 18}$ (P277L) (see Fig. 1A). However, it still remains to be analysed whether Prp31 proteins dimerize. As shown in yeast, Prp31 is a component of the spliceosomal U4/U6 di-SNP, which contains, beside the base-paired U4 and U6 snRNAs, more than 10 other proteins, including Prp3 and Prp4. In this complex, Prp31 is required to 
stabilize a U4/U6 snRNA junction, which in turn is required for binding of Prp3/4 (Hardin et al., 2015). In human PRPF31, the Nop domain is involved in an essential step in the formation of the U4/U6-U5 tri-snRNP by building a complex of the U4 snRNA and a $15.5 \mathrm{~K}$ protein. Consistent with this, many mutations in human PRPF31, which are linked to RP11, have been mapped to the Nop domain. Mutations in amino acid H270 in the Nop domain of human PRPF31 results in its reduced affinity to a complex formed by a stem-loop structure of the U4 snRNA and the $15.5 \mathrm{~K}$ protein (Liu et al., 2007; Schultz et al., 2006). Interestingly, the

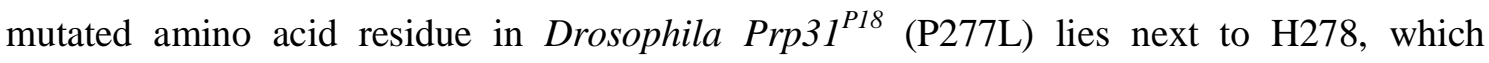
corresponds to amino acid H270 in the human protein. Therefore, it is tempting to speculate that the Drosophila P277L mutation could similarly weaken, but not abolish the corresponding interaction of the mutant Prp31 protein in flies, thus explaining the hypomorphic nature of this allele. Finally, PRC-specific RNAi-mediated knock-down of Prp31 induced a similar phenotype as the one observed in Prp31 heterozygous animals [data not shown and (Ray et al., 2010)]. The defects on eye morphogenesis detected upon more widespread knock-down of Prp31 (Ray et al., 2010) indicates that Prp31 may be important in other tissues as well, supporting our assumption that $\operatorname{Prp} 1^{P 17}$ and $\operatorname{Prp} 31^{P 18}$ are hypomorphic alleles. Further experiments are required to determine the functional consequence of the molecular lesions. Preliminary results make it unlikely that impaired nuclear localisation of the mutant proteins is responsible for the reduced function: the mutant proteins localise to nuclear speckles when expressed in HeLa cells (data not shown), and hence behave similar as the wild-type protein (Makarova et al., 2002).

Our results show that Prp31 heterozygous flies undergo retinal degeneration, a phenotype with striking similarity to human RP11 patients. This now allows to further unveil the cause of the aetiology of the disease in a model organism, which is easily accessible to genetic manipulations and molecular studies (Ugur et al., 2016). Our results propose a multifactorial genesis of Prp31-linked retinal degeneration in flies. i) We suggest that increased accumulation of intracellular rhodopsin may contribute to the degeneration in Prp31 mutant retinas. Immunostainings of retinae of hetero-, as well as homozygous $\operatorname{Prp}^{\text {P18 }}{ }^{18}$ flies showed an accumulation of Rh1 in the photoreceptor cell body in comparison to $w^{1118}$ control flies (data not shown). Accumulation of misfolded rhodopsin in the ER due to dominant mutations in the gene which impair the maturation of the protein, has been described to cause an overproduction of ER cisternae and eventually leads to degeneration (Colley et al., 1995). Interestingly, mis-localisation of rhodopsin in human PRCs to sites other than the outer segment is a common characteristic of various forms of RP and is considered to contribute to 
the pathological severity (Hollingsworth and Gross, 2012). Light-dependent PRC degeneration in Prp31-mutant flies could be prevented by raising mutant animals with food that lacks vitamin A, the precursor for retinal. This treatment reduces the amount of rhodopsin to about 3\% of its normal content (Nichols and Pak, 1985), and thus strongly reduces rhodopsin accumulation in the cell body.

ii) Our data further suggest that besides accumulation of intracellular rhodopsin increased oxidative stress contributes to light-dependent PRC degeneration in Prp31 mutant flies, since the degree of degeneration could be reduced by feeding flies with NSC23766, a known inhibitor of NADPH oxidase (NOX) activity. NADPH oxidases are multi-subunit enzyme complexes, comprising two membrane-bound and three cytoplasmic components, and can be found in the plasma-membrane of many cell types. One of the cytoplasmic components is the small GTPase Rac1 (Hordijk, 2006). Recruitment of the cytoplasmic components to the membrane activates the complex, resulting in transfer of electrons from NADPH to molecular oxygen, thus producing superoxide. NSC23766 selectively inhibits the interaction between Rac1 and Rac1-specific guanine nucleotide exchange factors (GEFs), thereby preventing the activation of Rac1 and hence NOX activity (Katsuyama, 2010; Rastogi et al., 2016). The suppression of retinal degeneration in Prp31 mutant flies by NSC23766 suggests that increased ROS production is causally related to degeneration. This assumption is supported by the observation that mutant flies show enhanced expression of GstD1-GFP, a reporter that serves as a proxy of ROS levels (Sykiotis and Bohmann, 2008).

Data obtained from transcriptome analysis of Prp31 mutant heads strongly support the conclusion that oxidative stress contributes to the mutant phenotype. The genes most highly upregulated in heads of Prp31 mutant flies match GO terms that are known to be upregulated upon oxidative stress (Girardot et al., 2006; Landis et al., 2004). These are, for example genes in involved in oxidation/reduction processes, e.g. desat2, Cytochrome P450-6a17 (Cyp6a17) and $C y p 9 c 1$. Some of the genes, which are downregulated in Prp31 mutant fly heads are those involved in polysaccharide/chitin metabolisms, e. g. Cht3, encoding a chitinase, and Tweedle $E$ (TwdlE), a cuticular protein (Cornman, 2009; Guan et al., 2006; Karouzou et al., 2007). Genes from this category were shown to be highly up-regulated in flies exposed to mild stress by increased atmospheric pressure (hyperbaric normoxia) (Yu et al., 2016). This stress has been suggested to induce cytoprotective responses, aimed to protect cells or organisms against the detrimental effects of stressors, such as senescence (Oh et al., 2008). Chitin oligosaccharides comprise a major constituent of the insect cuticle and have been suggested to 
function as antioxidants by scavenging ROS (Ngo and Kim, 2014) and to induce the immune response (Li et al., 2013). The downregulation of these genes in Prp31 mutant heads points to a reduced cytoprotective response and hence may contribute to the detrimental effects of light stress.

Taken together, the retina of Prp31 mutant flies exhibits increased oxidative stress response, suggesting that upon additional stress, provided by constant light exposure, the anti-oxidant response machinery is no longer able to prevent the damage induced by high levels of ROS. This may lead, among others, to oxidation of lipids, which are major constituents of the photosensitive organelle, the outer segments in vertebrates and the rhabdomeres in flies. Oxidised lipids may contribute to PRC degeneration, as shown for Age-related Macular Degeneration (AMD) (Handa et al., 2017).

iii) Data from the transcriptome analysis further reveal that impaired Prp31 function results in increased intron retention, suggesting defects in splicing. It is worth mentioning that with the exception of $A d h$, impaired splicing did not overlap with the down-regulated genes. From this we conclude that transcript down-regulation largely does not reflect nonsense-mediated mRNA decay, which often occurs in order to prevent translation of intron-containing mRNAs into non-functional and potentially detrimental proteins (Yap and Makeyev, 2013). Interestingly, genes with the most significant intron retention are those involved in regulating the function, localisation and maturation of synaptic vesicles. Whether this results in defective synaptic transmission, remains to be elucidated. Previous work has shown that mutations in genes required for proper synapse organization in PRCs may eventually result in retinal degeneration, e. g mutations in Drosophila Lin-7/veli (Soukup et al., 2013). Similarly, mislocalization of pre- and postsynaptic proteins, as observed in $r d l$ and $r d 10$ mutant mice [reviewed in (Soto and Kerschensteiner, 2015)], or structural abnormalities in PRC synapses as observed in tulp mutant mice (Grossman et al., 2009) precede PRC degeneration.

Taken together, using the fly as a genetic model revealed a multifactorial genesis of lightdependent retinal degeneration of Prp31 mutant flies. Impaired Prp31 function impacts on various cellular processes and results in increased oxidative stress, defective rhodopsin transport/maturation and intron retention, predominantly in transcripts of genes involved in synapse formation and function. To what extend these dysfunctions influence each other has to be elucidated. 


\section{Materials and Methods}

Fly maintenance and genetics: Flies and crosses were maintained at $25^{\circ} \mathrm{C}$, on standard yeast-cornmeal-agar food, under 12 hours of light/12 hours of darkness, unless specified. Flies were placed for a total of 7 days under these conditions. Genetic control for all experiments was white $\left(w^{*}\right)$. Deficiency lines used here were obtained from the Bloomington Stock Centre and included $D f(3 L)$ Exel6262 (Parks et al., 2004), Df(3L)ED217and Df(3L)ED218 (Ryder et al., 2007). GstD-GFP flies (Sykiotis and Bohmann, 2008) (gift from D. Bohman) were used as an indicator of oxidative stress signalling by combining into the $\operatorname{Prp} 31^{18}$ genetic background or into the genetic control $\left(w^{*}\right)$. For the light-stress experimental paradigm, flies were kept at $25^{\circ} \mathrm{C}$ for 7 days in a special incubator designed to have high intensity (1200-1300 lux), continuous light exposure (Johnson et al., 2002)

Anti-oxidant feeding: Female flies were collected immediately upon eclosion and divided into groups maintained on regular food, supplemented with a Whatman® filter paper (GE Healthcare) soaked in 5\% sucrose (control) or with $500 \mu \mathrm{M}$ NSC 23766 (Cayman Chemical). Food and paper were changed every second day.

Vitamin A depletion: For vitamin A depletion experiments, flies were raised from embryonic stages until adulthood and subsequently maintained on carotenoid free food (10\% dry yeast, $10 \%$ sucrose, $0.02 \%$ cholesterol, and $2 \%$ agar) as described (Pocha et al., 2011).

Hydrogen Peroxide exposure: Flies were raised on standard yeast-food and upon eclosion, were transferred in groups of 10 onto standard food or food supplemented with $5 \% \mathrm{H}_{2} \mathrm{O}_{2}$ (Sigma-Aldrich, Germany). After two days under 12h light and 12h dark conditions, 4-6 flies/genotype were used for further analyses

Quantification of GstD-GFP following $\mathrm{H}_{2} \mathrm{O}_{2}$ feeding: Flies were lysed in $200 \mu$ l of phosphate buffer ( $\mathrm{pH}$ 7.4-7.6) with $0.1 \%$ Tween-20 on ice. Lysates were centrifuged at $15.000 \mathrm{rpm}$ for 10 minutes. Of this, $25 \mu \mathrm{l}$ was used to estimate protein content by BCA assay and $150 \mu \mathrm{l}$ was used for fluorescence measurements using a Plate reader (Perkin Elmer Envision) and 485nm excitation \& 590-10nm Emission filters. To calculate percent change, fluorescence values were normalized after arbitrarily setting values of control flies ( $g s t D$ $G F P /+$ ) at 100. Data from 5 biological replicates (standard food) and 2 biological replicates 
$\left(5 \% \mathrm{H}_{2} \mathrm{O}_{2}\right)$ were used for the analyses. ANOVA and post-hoc Bonferroni test was used to compare different samples.

Isolation of Prp31 alleles by TILLING: To isolate point mutations in the Prp31 locus (FlyBase ID: FBgn0036487) a library, of 2.400 fly lines with isogenized third chromosomes which potentially carry point mutations caused by EMS treatment, was screened. Our approach targeted exon 1-3 of the Prp31 locus containing two thirds (67\%) of the coding sequence and including several predicted functional domains (the NOSIC (IPRO012976), the Nop (IPRO002687) and parts of the Prp31_C terminal (IPRO019175) domain), making use of two different PCR amplicons. A nested PCR approach was used, where the inner primers contain universal M13 tails that serve as primer binding sites of the Sanger sequencing reaction:

- amplicon1 (covers exon 1 and 2), outer primer, forward: TTCAATGAACCGCATGG, reverse: GTCGATCTTTGCCTTCTCC, inner / nested primer, forward: TGTAAAACGA CGGCCAGT-AGCAACGGTCACTTCAATTC， reverse: AGGAAACAGCTATGACCAT-GAAAGGGAATGGGATTCAG);

- amplicon 2 (covers exon 3), outer primer, forward: ATCGTGGGTGAAATCGAG, reverse: TGGTCTTCTCATCCACCTG, inner / nested primer, forward: TGTAAAACGA CGGCCAGT-AAGCTGCAGGCTATTCTCAC, reverse: AGGAAACAGCTATGACCAT-TAGGCATCCTCTTCGATCTG.

PCR-reactions were performed in $10 \mu \mathrm{l}$ volume and with an annealing temperature of $57{ }^{\circ} \mathrm{C}$, in 384 well format, making use of automated liquid handling tools. PCR fragments were sequenced by Sanger sequencing optimized for amplicon re-sequencing in a large-scale format (Winkler et al., 2011; Winkler et al., 2005). Primary hits, resembling sequence variants, which upon translation result in potential nonsense and missense mutations or affect a predicted splice site, were verified in an independent PCR amplification and Sanger sequencing reaction.

Transmission electron microscopy: Fixation of adult eyes, semi-thin sections and ultra-thin sections for transmission electron microscopy was performed as described (Mishra and Knust, 2013). $2 \mu \mathrm{m}$ semi-thin sections were stained with a toluidine blue $1 \%$ / sodium tetraborate dehydrate $0.5 \%$ solution and imaged with AxioImager.Z1 (Zeiss, Germany) with an AxioCamMRm and the AxioVision software (Release 4.7). 70nm ultrathin sections were 
imaged using a Morgagni 268 TEM (100kV) electron microscope (FEI Company), and images were taken using a Side-entry Morada CCD Camera (11 Megapixels, Olympus).

Quantification of Degeneration: Quantification of degeneration was performed as described in (Bulgakova et al., 2010). Briefly, from the semi-thin sections, the number of outer photoreceptor cells (R1-R6) with clearly detectable rhabdomeres in each ommatidium were recorded as surviving rhabdomeres. In each section, 50-60 ommatidia were counted and for each genotype, 6 eyes from different individuals were analysed, unless indicated. ANOVA and post-hoc Bonferroni correction and two-tailed Mann-Whitney U test, respectively, were used to compare different distributions of photoreceptor cell survival.

Cryosections of Drosophila eyes: Adult eyes were dissected and fixed in $4 \%$ formaldehyde. Following sucrose treatment and embedding of the tissues in Richard-Allan Scientific NEG$50^{\mathrm{TM}}$ (Thermo Fisher Scientific, UK) tissue embedding medium, tissues were cryosectioned at $10 \mu \mathrm{m}$ thickness at $-21^{\circ} \mathrm{C}$. Sections were air-dried and then subjected to immunostaining as described previously (Spannl et al., 2017). Antibodies used were rabbit anti-GFP (1:500; A11122; Thermo Fisher Scientific, UK) and mouse anti-Na ${ }^{+}-\mathrm{K}^{+}$-ATPase (1:100; a5; Developmental Studies Hybridoma Bank, University of Iowa, USA). Alexa-Flour conjugated secondary antibodies (Thermo Fisher Scientific, UK) were used. F-actin was visualised with Alexa-Fluor-555-phalloidin (Thermo Fisher Scientific, UK). Images were taken on Olympus FV100 and processed using ImageJ/Fiji, Adobe Photoshop CS5.1 and Adobe Illustrator CS3 for image assembly.

RNA extraction: Whole RNA was extracted from fly heads (2 days old) with the RNeasy Mini Kit (Qiagen) according to the manufacturer's instructions. Each biological replicate consisted of 50 heads. A total of 3 biological replicates were used for further analyses.

RNA-seq and analyses: RNA-Seq was carried out in triplicate by the Deep Sequencing Group SFB 655 at Biotechnology Center, TU Dresden, with 76-bp single read sequencing on the Illumina HiSeq 2500. An average of 28.85 million reads per sample was obtained. Data analysis was carried out by the Scientific Computing Facility (MPI-CBG) by mapping to the *Drosophila* genome (BDGP6, Ensembl v81) using STAR (v2.5.1b). The differential expression analysis was performed using DESeq2 using a lfcThreshold parameter of 1.0 and Independent Hypothesis Weighting (Love et al., 2014). 115 differentially expressed genes were obtained with a q-value cutoff of 0.01 . Detection of differentially spliced genes was carried out using the DEXSeq Bioconductor package (Anders et al., 2012) with a discovery 
rate (FDR) threshold of 0.1. To test for intron retention as well as for differential exon usage, the DEXSeq model used to estimate exon abundance was complemented with intron regions (similar to the method presented by (Sauerwald et al., 2017)). clusterProfiler (Yu et al., 2012) was used to identify enrichment on a 0.05 q-value level for biological processes and pathways associated with the up- and down-regulated genes and for genes whose introns are differentially retained. The transcriptome data have been submitted to GEO. The accession number is GSE99665.

Figure panel preparation: All figure panels were assembled using Adobe Illustrator CS3 and Inkscape. Statistical analyses and graphs were generated using GraphPad Prism (GraphPad Software, Inc, USA) and Microsoft Excel. For protein sequence visualization, Illustrator of Biological Sequences (IBS; (Liu et al., 2015)) software package was used.

\section{References}

Anders, S., Reyes, A. and Huber, W. (2012). Detecting differential usage of exons from RNA-seq data. Genome research 22, 2008-2017.

Balasubramanian, N. and Slepak, V. Z. (2003). Light-mediated activation of Rac-1 in photoreceptor outer segments. Curr Biol 13, 1306-1310.

Bedard, K. and Krause, K. H. (2007). The NOX family of ROS-generating NADPH oxidases: physiology and pathophysiology. Physiol Rev 87, 245-313.

Belmonte, M. A., Santos, M. F., Kihara, A. H., Yan, C. Y. and Hamassaki, D. E. (2006). Light-Induced photoreceptor degeneration in the mouse involves activation of the small GTPase Rac1. Investigative ophthalmology \& visual science 47, 1193-1200.

Bujakowska, K., Maubaret, C., Chakarova, C. F., Tanimoto, N., Beck, S. C., Fahl, E., Humphries, M. M., Kenna, P. F., Makarov, E., Makarova, O. et al. (2009). Study of gene-targeted mouse models of splicing factor gene Prpf31 implicated in human autosomal dominant retinitis pigmentosa (RP). Investigative ophthalmology \& visual science 50, 5927-5933.

Bulgakova, N. A., Rentsch, M. and Knust, E. (2010). Antagonistic functions of two Stardust isoforms in Drosophila photoreceptor cells. Mol Biol Cell 21, 3915-3925.

Chartier, F. J.-M., Hardy , E. J.-L. and Laprise, P. (2012). Crumbs limits oxidasedependent signaling to maintain epithelial integrity and prevent photoreceptor cell death. $J$ Cell Biol 198, 991-998.

Colley, N. J., Cassill, J. A., Baker, E. K. and Zuker, C. S. (1995). Defective intracellular transport is the molecular basis of rhodopsin-dependent dominant retinal degeneration. Proc Natl Acad Sci U S A 92, 3070-3074.

Cornman, R. S. (2009). Molecular evolution of Drosophila cuticular protein genes. PLoS One 4, e8345.

Daiger, S. P., Bowne, S. J. and Sullivan, L. S. (2014). Genes and Mutations Causing Autosomal Dominant Retinitis Pigmentosa. Cold Spring Harb Perspect Med $\mathbf{5}$.

Farkas, M. H., Lew, D. S., Sousa, M. E., Bujakowska, K., Chatagnon, J., Bhattacharya, S. S., Pierce, E. A. and Nandrot, E. F. (2014). Mutations in pre-mRNA processing factors 3,8 , and 31 cause dysfunction of the retinal pigment epithelium. Am J Pathol 184, 2641-2652. 
Gepner, J., Li, M., Ludmann, S., Kortas, C., Boylan, K., Iyadurai, S. J., McGrail, M. and Hays, T. S. (1996). Cytoplasmic dynein function is essential in Drosophila melanogaster. Genetics 142, 865-878.

German, O. L., Agnolazza, D. L., Politi, L. E. and Rotstein, N. P. (2015). Light, lipids and photoreceptor survival: live or let die? Photochemical \& photobiological sciences : Official journal of the European Photochemistry Association and the European Society for Photobiology 14, 1737-1753.

Girardot, F., Lasbleiz, C., Monnier, V. and Tricoire, H. (2006). Specific agerelated signatures in Drosophila body parts transcriptome. BMC Genomics 7, 69.

Graziotto, J. J., Farkas, M. H., Bujakowska, K., Deramaudt, B. M., Zhang, Q., Nandrot, E. F., Inglehearn, C. F., Bhattacharya, S. S. and Pierce, E. A. (2011). Three gene-targeted mouse models of RNA splicing factor RP show late-onset RPE and retinal degeneration. Investigative ophthalmology \& visual science 52, 190-198.

Grossman, G. H., Pauer, G. J., Narendra, U., Peachey, N. S. and Hagstrom, S. A. (2009). Early synaptic defects in tulp1-/- mice. Investigative ophthalmology \& visual science 50, 3074-3083.

Guan, X., Middlebrooks, B. W., Alexander, S. and Wasserman, S. A. (2006). Mutation of TweedleD, a member of an unconventional cuticle protein family, alters body shape in Drosophila. Proc Natl Acad Sci U S A 103, 16794-1679.

Handa, J. T., Cano, M., Wang, L., Datta, S. and Liu, T. (2017). Lipids, oxidized lipids, oxidation-specific epitopes, and Age-related Macular Degeneration. Biochim Biophys Acta 1862, 430-440.

Hardin, J. W., Warnasooriya, C., Kondo, Y., Nagai, K. and Rueda, D. (2015). Assembly and dynamics of the U4/U6 di-snRNP by single-molecule FRET. Nucleic acids research 43, 10963-10974.

Haruta, M., Bush, R. A., Kjellstrom, S., Vijayasarathy, C., Zeng, Y., Le, Y. Z. and Sieving, P. A. (2009). Depleting Rac1 in mouse rod photoreceptors protects them from photo-oxidative stress without affecting their structure or function. Proc Natl Acad Sci US A 106, 9397-9402.

Hollingsworth, T. J. and Gross, A. K. (2012). Defective trafficking of rhodopsin and its role in retinal degenerations. International review of cell and molecular biology 293, 1-44.

Hordijk, P. L. (2006). Regulation of NADPH oxidases: the role of Rac proteins. Circ Res 98, 453-62.

Johnson, K., Grawe, F., Grzeschik, N. and Knust, E. (2002). Drosophila Crumbs is required to inhibit light-induced photoreceptor degeneration. Curr Biol 12, 1675-1680.

Jones, R. M., Luo, L., Ardita, C. S., Richardson, A. N., Kwon, Y. M., Mercante, J. W., Alam, A., Gates, C. L., Wu, H., Swanson, P. A. et al. (2013). Symbiotic lactobacilli stimulate gut epithelial proliferation via Nox-mediated generation of reactive oxygen species. Embo J 32, 3017-3028.

Karouzou, M. V., Spyropoulos, Y., Iconomidou, V. A., Cornman, R. S., Hamodrakas, S. J. and Willis, J. H. (2007). Drosophila cuticular proteins with the R\&R Consensus: annotation and classification with a new tool for discriminating RR-1 and RR-2 sequences. Insect Biochem Mol Biol 37, 754-760.

Katsuyama, M. (2010). NOX/NADPH oxidase, the superoxide-generating enzyme: its transcriptional regulation and physiological roles. J Pharmacol Sci 114, 134-146.

Landis, G. N., Abdueva, D., Skvortsov, D., Yang, J., Rabin, B. E., Carrick, J., Tavare, S. and Tower, J. (2004). Similar gene expression patterns characterize aging and oxidative stress in Drosophila melanogaster. Proc Natl Acad Sci U S A 101, 7663-7668.

Li, X., Min, M., Du, N., Gu, Y., Hode, T., Naylor, M., Chen, D., Nordquist, R. E. and Chen, W. R. (2013). Chitin, chitosan, and glycated chitosan regulate immune responses: the novel adjuvants for cancer vaccine. Clin Dev Immunol 2013, 387023. 
Linder, B., Dill, H., Hirmer, A., Brocher, J., Lee, G. P., Mathavan, S., Bolz, H. J., Winkler, C., Laggerbauer, B. and Fischer, U. (2011). Systemic splicing factor deficiency causes tissue-specific defects: a zebrafish model for retinitis pigmentosa. Hum Mol Genet 20, 368-377.

Liu, M. M. and Zack, D. J. (2013). Alternative splicing and retinal degeneration. Clin Genet 84, 142-149.

Liu, S., Li, P., Dybkov, O., Nottrott, S., Hartmuth, K., Luhrmann, R., Carlomagno, T. and Wahl, M. C. (2007). Binding of the human Prp31 Nop domain to a composite RNA-protein platform in U4 snRNP. Science 316, 115-120.

Liu, W., Xie, Y., Ma, J., Luo, X., Nie, P., Zuo, Z., Lahrmann, U., Zhao, Q., Zheng, Y., Zhao, Y. et al. (2015). IBS: an illustrator for the presentation and visualization of biological sequences. Bioinformatics 31, 3359-3361.

Love, M. I., Huber, W. and Anders, S. (2014). Moderated estimation of fold change and dispersion for RNA-seq data with DESeq2. Genome biology 15, 550.

Maita, H., Kitaura, H., Keen, T. J., Inglehearn, C. F., Ariga, H. and Iguchi-Ariga, S. M. (2004). PAP-1, the mutated gene underlying the RP9 form of dominant retinitis pigmentosa, is a splicing factor. Exp Cell Res 300, 283-296.

Makarova, O. V., Makarov, E. M., Liu, S., Vornlocher, H. P. and Luhrmann, R. (2002). Protein 61K, encoded by a gene (PRPF31) linked to autosomal dominant retinitis pigmentosa, is required for U4/U6*U5 tri-snRNP formation and pre-mRNA splicing. Embo $J$ 21, 1148-1157.

Mi, H., Muruganujan, A. and Thomas, P. D. (2013). PANTHER in 2013: modeling the evolution of gene function, and other gene attributes, in the context of phylogenetic trees. Nucleic acids research 41, D377-386.

Mordes, D., Luo, X., Kar, A., Kuo, D., Xu, L., Fushimi, K., Yu, G., Sternberg, P., Jr. and Wu, J. Y. (2006). Pre-mRNA splicing and retinitis pigmentosa. Mol Vis 12, 12591271.

Nassar, N., Cancelas, J., Zheng, J., Williams, D. A. and Zheng, Y. (2006). Structure-function based design of small molecule inhibitors targeting Rho family GTPases. Curr Top Med Chem 6, 1109-1116.

Ng, S. K., Wood, J. P., Chidlow, G., Han, G., Kittipassorn, T., Peet, D. J. and Casson, R. J. (2015). Cancer-like metabolism of the mammalian retina. Clin Exp Ophthalmol 43, 367-376.

Ngo, D. H. and Kim, S. K. (2014). Antioxidant effects of chitin, chitosan, and their derivatives. Adv Food Nutr Res 73, 15-31.

Nichols, R. and Pak, W. L. (1985). Characterization of Drosophila melanogaster rhodopsin. J Biol Chem 260, 12670-12674.

Oh, S., Lee, E., Lee, J., Lim, Y., Kim, J. and Woo, S. (2008). Comparison of the effects of $40 \%$ oxygen and two atmospheric absolute air pressure conditions on stress-induced premature senescence of normal human diploid fibroblasts. Cell Stress Chaperones 13, 447458.

Parks, A. L., Cook, K. R., Belvin, M., Dompe, N. A., Fawcett, R., Huppert, K., Tan, L. R., Winter, C. G., Bogart, K. P., Deal, J. E. et al. (2004). Systematic generation of high-resolution deletion coverage of the Drosophila melanogaster genome. Nat Genet 36, 288-292.

Pocha, S. M., Shevchenko, A. and Knust, E. (2011). Crumbs regulates rhodopsin transport by interacting with and stabilizing myosin V. J Cell Biol 195, 827-838.

Poulos, M. G., Batra, R., Charizanis, K. and Swanson, M. S. (2011). Developments in RNA splicing and disease. Cold Spring Harbor perspectives in biology 3, a000778. 
Punzo, C., Xiong, W. and Cepko, C. L. (2012). Loss of daylight vision in retinal degeneration: are oxidative stress and metabolic dysregulation to blame? J Biol Chem 287, 1642-1648.

Rastogi, R., Geng, X., Li, F. and Ding, Y. (2016). NOX Activation by Subunit Interaction and Underlying Mechanisms in Disease. Front Cell Neurosci 10, 301.

Ray, P., Luo, X., Rao, E. J., Basha, A., Woodruff, E. A., 3rd and Wu, J. Y. (2010). The splicing factor Prp31 is essential for photoreceptor development in Drosophila. Protein Cell 1, 267-274.

Rio Frio, T., Wade, N. M., Ransijn, A., Berson, E. L., Beckmann, J. S. and Rivolta, C. (2008). Premature termination codons in PRPF31 cause retinitis pigmentosa via haploinsufficiency due to nonsense-mediated mRNA decay. J Clin Invest 118, 1519-1531.

Ruzickova, S. and Stanek, D. (2016). Mutations in spliceosomal proteins and retina degeneration. RNA Biol, 1-9.

Ryder, E., Ashburner, M., Bautista-Llacer, R., Drummond, J., Webster, J., Johnson, G., Morley, T., Chan, Y. S., Blows, F., Coulson, D. et al. (2007). The DrosDel deletion collection: a Drosophila genomewide chromosomal deficiency resource. Genetics 177, 615-629.

Sauerwald, J., Soneson, C., Robinson, M. D. and Luschnig, S. (2017). Faithful mRNA splicing depends on the Prp19 complex subunit faint sausage and is required for tracheal branching morphogenesis in Drosophila. Development 144, 657-663.

Schultz, A., Nottrott, S., Hartmuth, K. and Luhrmann, R. (2006). RNA structural requirements for the association of the spliceosomal hPrp31 protein with the U4 and U4atac small nuclear ribonucleoproteins. J Biol Chem 281, 28278-28286.

Scotti, M. M. and Swanson, M. S. (2016). RNA mis-splicing in disease. Nat Rev Genet 17, 19-32.

Song, H., Vijayasarathy, C., Zeng, Y., Marangoni, D., Bush, R. A., Wu, Z. and Sieving, P. A. (2016). NADPH Oxidase Contributes to Photoreceptor Degeneration in Constitutively Active RAC1 Mice. Investigative ophthalmology \& visual science 57, 28642875.

Soto, F. and Kerschensteiner, D. (2015). Synaptic remodeling of neuronal circuits in early retinal degeneration. Front Cell Neurosci 9, 395.

Soukup, S. F., Pocha, S. M., Yuan, M. and Knust, E. (2013). DLin-7 is required in postsynaptic lamina neurons to prevent light-induced photoreceptor degeneration in Drosophila. Curr Biol 23, 1349-1354.

Spannl, S., Kumichel, A., Hebbar, S., Kapp, K., Gonzalez-Gaitan, M., Winkler, S., Blawid, R., Jessberger, G. and Knust, E. (2017). The Crumbs_C isoform of Drosophila shows tissue- and stage-specific expression and prevents light-dependent retinal degeneration. Biol Open 6, 165-175.

Sykiotis, G. P. and Bohmann, D. (2008). Keap1/Nrf2 signaling regulates oxidative stress tolerance and lifespan in Drosophila. Dev Cell 14, 76-85.

Tanackovic, G., Ransijn, A., Thibault, P., Abou Elela, S., Klinck, R., Berson, E. L., Chabot, B. and Rivolta, C. (2011). PRPF mutations are associated with generalized defects in spliceosome formation and pre-mRNA splicing in patients with retinitis pigmentosa. Hum Mol Genet 20, 2116-2130.

Thomas, P. D., Campbell, M. J., Kejariwal, A., Mi, H., Karlak, B., Daverman, R., Diemer, K., Muruganujan, A. and Narechania, A. (2003). PANTHER: a library of protein families and subfamilies indexed by function. Genome research 13, 2129-2141.

Tomanek, L. (2015). Proteomic responses to environmentally induced oxidative stress. J Exp Biol 218, 1867-1879.

Ugur, B., Chen, K. and Bellen, H. J. (2016). Drosophila tools and assays for the study of human diseases. Dis Model Mech 9, 235-244. 
Will, C. L. and Luhrmann, R. (2011). Spliceosome structure and function. Cold Spring Harbor perspectives in biology 3, pii: a003707.

Winkler, S., Gscheidel, N. and Brand, M. (2011). Mutant generation in vertebrate model organisms by TILLING. Methods Mol Biol 770, 475-504.

Winkler, S., Schwabedissen, A., Backasch, D., Bokel, C., Seidel, C., Bonisch, S., Furthauer, M., Kuhrs, A., Cobreros, L., Brand, M. et al. (2005). Target-selected mutant screen by TILLING in Drosophila. Genome research 15, 718-723.

Wu, C. T. and Howe, M. (1995). A genetic analysis of the Suppressor 2 of zeste complex of Drosophila melanogaster. Genetics 140, 139-181.

Yap, K. and Makeyev, E. V. (2013). Regulation of gene expression in mammalian nervous system through alternative pre-mRNA splicing coupled with RNA quality control mechanisms. Molecular and cellular neurosciences 56, 420-428.

Yin, J., Brocher, J., Fischer, U. and Winkler, C. (2011). Mutant Prpf31 causes premRNA splicing defects and rod photoreceptor cell degeneration in a zebrafish model for Retinitis pigmentosa. Mol Neurodegener 6, 56.

Yu, D. Y. and Cringle, S. J. (2001). Oxygen distribution and consumption within the retina in vascularised and avascular retinas and in animal models of retinal disease. Prog Retin Eye Res 20, 175-208.

Yu, G., Wang, L. G., Han, Y. and He, Q. Y. (2012). clusterProfiler: an R package for comparing biological themes among gene clusters. OMICS 16, 284-287.

Yu, S., Lee, E., Tsogbadrakh, B., Son, G. I. and Kim, M. (2016). Prenatal hyperbaric normoxia treatment improves healthspan and regulates chitin metabolic genes in Drosophila melanogaster. Aging (Albany NY) 8, 2538-2550.

Yuan, L., Kawada, M., Havlioglu, N., Tang, H. and Wu, J. Y. (2005). Mutations in PRPF31 inhibit pre-mRNA splicing of rhodopsin gene and cause apoptosis of retinal cells. $J$ Neurosci 25, 748-757.

Zhao, C., Bellur, D. L., Lu, S., Zhao, F., Grassi, M. A., Bowne, S. J., Sullivan, L. S., Daiger, S. P., Chen, L. J., Pang, C. P. et al. (2009). Autosomal-dominant retinitis pigmentosa caused by a mutation in SNRNP200, a gene required for unwinding of U4/U6 snRNAs. Am J Hum Genet 85, 617-627. 


\section{Figure Legends}

Figure 1: Prp31 mutant flies have no gross morphological abnormalities at eclosion.

(A) Schematic overview of the human Prpf31 protein. The figure is drawn to scale using IBS (Liu et al., 2015). Domains and TILLING mutations described here are indicated.

(B-E') are representative electron micrographs of 70nm sections of eyes of genetic control $w^{*}$ (B, B'), $\operatorname{Prp} 31^{P 17} /+\left(\mathrm{C}, \mathrm{C}^{\prime}\right), \operatorname{Prp} 31^{P 18} /+\left(\mathrm{D}, \mathrm{D}^{\prime}\right)$, and $\operatorname{Prp} 31^{P 18} / \operatorname{Prp} 31^{P 18}$ (E, E'). Upon eclosion, flies were kept for two days under regular light conditions. B', C', D' and E' depict higher magnifications of one ommatidium of the respective overviews shown in B, C, D and E.

Note that the number and stereotypic arrangement of photoreceptor cells within the mutant ommatidia are not affected. Scale bars represent $10 \mu \mathrm{m}$ in B, C, D and E and $1 \mu \mathrm{m}$ in B', C', D' and E'.

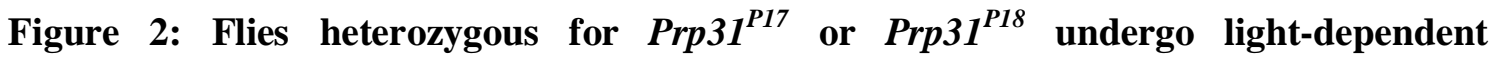 degeneration.}

(A-C') are representative electron micrographs of 70nm sections of eyes of genetic control $w^{*}$ (A, A'), $\operatorname{Prp} 31^{P 17} /+\left(\mathrm{B}, \mathrm{B}^{\prime}\right), \operatorname{Prp} 31^{P 18} /+(\mathrm{C}, \mathrm{C})$ exposed for 7 days to continuous, high intensity light. (D-E') are representative electron micrographs of $\operatorname{Prp} 31^{P 17} / \operatorname{Prp} 31^{P 18}$ eyes exposed to regular light conditions (12 hours light/12 hours dark; D, D') and continuous high intensity light (E, E'). Note that degeneration under constant light exposure is more pronounced in Prp31 heterozygotes (B, B' and C, C') than in transheterozyotes (E, E'). The most obvious features of degenerating PRCs are (i) absence (yellow arrowhead) or reduction (yellow arrow) of rhabdomeres of outer photoreceptor cells (R1-R6), (ii) accumulation of electron dense material with vacuolization (red arrowhead) as seen in B' and C'. Transheterozygotes do not show any defect when kept under regular light conditions (D, D'), but exhibit loss of rhabdomeric integrity (yellow asterisks), which is considered as an early sign of degeneration, upon exposure to constant light (E, E'), Scale bars represent $10 \mu \mathrm{m}$ in $\mathrm{A}, \mathrm{B}, \mathrm{C}, \mathrm{D}$ and $\mathrm{E}$ and $1 \mu \mathrm{m}$ in $\mathrm{A}^{\prime}, \mathrm{B}^{\prime}, \mathrm{C}^{\prime}, \mathrm{D}^{\prime}$ and $\mathrm{E}$ '.

(F) Quantification of retinal degeneration. Bars represent mean \pm s.e.m. for the number of surviving rhabdomeres of outer PRCs R1-R6 following high intensity, continuous light 
bioRxiv preprint doi: https://doi.org/10.1101/147918; this version posted June 9, 2017. The copyright holder for this preprint (which was not certified by peer review) is the author/funder. All rights reserved. No reuse allowed without permission.

exposure. Significant differences are indicated by black horizontal bars. *** indicates significance at $\mathrm{p}<0.005$ calculated using ANOVA and post-hoc Bonferroni correction. 


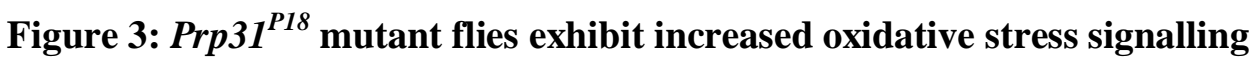

A: Percent GFP fluorescence levels measured from lysates of adult flies of the indicated genotypes after normalization to fluorescence levels in control flies $(g s t D-G F P /+)$, set at 100 (arbitrary units). Flies were fed on a standard yeast diet or on standard yeast food supplemented with $5 \% \mathrm{H}_{2} \mathrm{O}_{2}$. The values were averaged from 5 biological replicates (standard food) and 2 biological replicates $\left(5 \% \mathrm{H}_{2} \mathrm{O}_{2}\right)$. Comparisons are indicated by the black horizontal bars, * indicates significance at $\mathrm{p}<0.05$, *** indicates significance at $\mathrm{p}<0.005$ and ns indicates non-significance calculated using ANOVA followed by a post-hoc Bonferroni correction. Bars represent mean \pm s.e.m.

B-C': Top is a cartoon of an ommatidium including the 7 photoreceptor cells (R1-R7) with their photosensitive organelle called the rhabdomere (magenta). Pigment cells (pc) lie adjacent to the photoreceptor cells. Bottom are representative $1 \mu \mathrm{m}$ confocal optical sections imaged from a $10 \mu \mathrm{m}$ section of an eye of GstD-GFP;+ (B, B') or GstD-GFP; Prp31 $1^{\text {P18 }}$ heterozygotes (C, C'). Sections have been labelled with anti-GFP (green; GstD activity), phalloidin (magenta; rhabdomeres) and anti- $\mathrm{Na}^{+}-\mathrm{K}^{+}$-ATPase (white; basal membranes of photoreceptor cells). Boxed outlines in $\mathrm{B}$ and $\mathrm{C}$ indicate the ommatidium shown at higher magnification in B' and C', respectively. In control eyes (B, B'), GFP is evident in cone cells (cc) and in the pigment cells (pc) surrounding each ommatidium. Gst-GFP expression is increased in $\operatorname{Prp} 31^{P 18}$ mutants $\left(\mathrm{C}, \mathrm{C}^{\prime}\right)$. Note that in contrast to high GFP levels in support cells, no GFP was detected in photoreceptor cells.

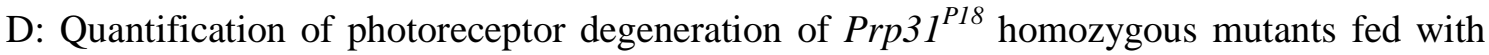
5\% sucrose or with $500 \mu \mathrm{M}$ NSC 23766 in 5\% sucrose. Graph represents mean \pm s.e.m. of surviving rhabdomeres of outer PRCs R1-R6, measured after 7 days exposure to constant, high intensity light. Feeding with NSC 23766 significantly reduced degeneration. *** $\mathrm{p}<0.005$ as determined by a two-tailed Mann-Whitney U test.

\section{Figure 4: Raising Prp31 mutant animals in vitamin A-depleted food rescues light- dependent degeneration.}

(A-B') are representative electron micrographs of 70nm sections of eyes of $\operatorname{Prp} 31^{P 17} /+$ (A, A') and $\operatorname{Prp} 31^{P 18} /+$ (B, B') raised on vitamin A deficient food and placed in constant, high intensity light for seven days. Rhabdomeres are smaller due to vitamin A deficiency, but 
overall there are no obvious signs of retinal degeneration (compare to Fig. 2C, C'). Scale bars represent $10 \mu \mathrm{m}$ in $\mathrm{A}$ and $\mathrm{B}$ and $1 \mu \mathrm{m}$ in $\mathrm{A}^{\prime}$ and $\mathrm{B}^{\prime}$

(C) Quantification of photoreceptor degeneration in Prp31 heterozygous mutants raised with normal and with vitamin A-depleted food. Bars represent mean \pm s.e.m. of surviving rhabdomeres of outer PRCs R1-R6 in $P r p 31^{P 17} /+$ and $\operatorname{Prp} 31^{P 18} /+$ flies raised and kept on vitamin A deficient food after seven days of exposure to constant light. ** indicates significance at $\mathrm{p}<0.01$ calculated using a two-tailed Mann-Whitney $\mathrm{U}$ test.

\section{Figure 5: Classification of Up- and Down-regulated genes in $\operatorname{Prp}{ }^{P 18}$ fly heads}

(A-B) Pie charts showing the classification of the 52 genes with significantly increased (A) and the 63 genes with significantly decreased (B) expression in mutant $\operatorname{Prp} 31^{P 18} / \operatorname{Prp} 31^{P 18}$ heads, compared to control heads using the Gene Ontology/PANTHER classification system, based on the predicted protein classes for their gene products. Colors represent the different categories in the pie charts.

(C) Bars show the significantly enriched GO terms for all genes that are differentially expressed in the mutant, compared to the genetic control, classified according to the GO category "molecular function". The Y-axis indicates the sub-categories, the X-axis indicates the significance of the enrichment in GO terms compared to genes in total. Gene symbols of the genes falling into the category are stated in the bars.

\section{Figure 6: Classification of retained introns in $\operatorname{Prp} 31^{P 18}$ mutants}

Bars show the significantly enriched GO terms for genes that display intron retention in the mutant, compared to the genetic control, classified according to the GO category "biological process" ( $\mathrm{p}$-value $\leq 1.12 \mathrm{E}-04$ ). The $\mathrm{Y}$-axis indicates the sub-categories, the $\mathrm{X}$-axis indicates the significance of the enrichment in GO terms compared to genes in total. Gene symbols of the genes falling into the individual categories are listed.

Supplementary Figure 1: Amino acid sequence comparison of Drosophila Prp31 (upper) and human PRPF31 protein (lower). The NOSIC (yellow), Nop (red) and Prp31_C specific (green) domains are indicated as described on UniProt and Pfam websites. TILLING mutations are indicated in magenta. Asterisks (*) indicate fully conserved amino acids, colons 
bioRxiv preprint doi: https://doi.org/10.1101/147918; this version posted June 9, 2017. The copyright holder for this preprint (which was not certified by peer review) is the author/funder. All rights reserved. No reuse allowed without permission.

(:) indicate groups of amino acids of strongly similar properties and periods (.) indicate amino acids with weakly similar properties. Alignment was made using ClustalO 1.2.3. 

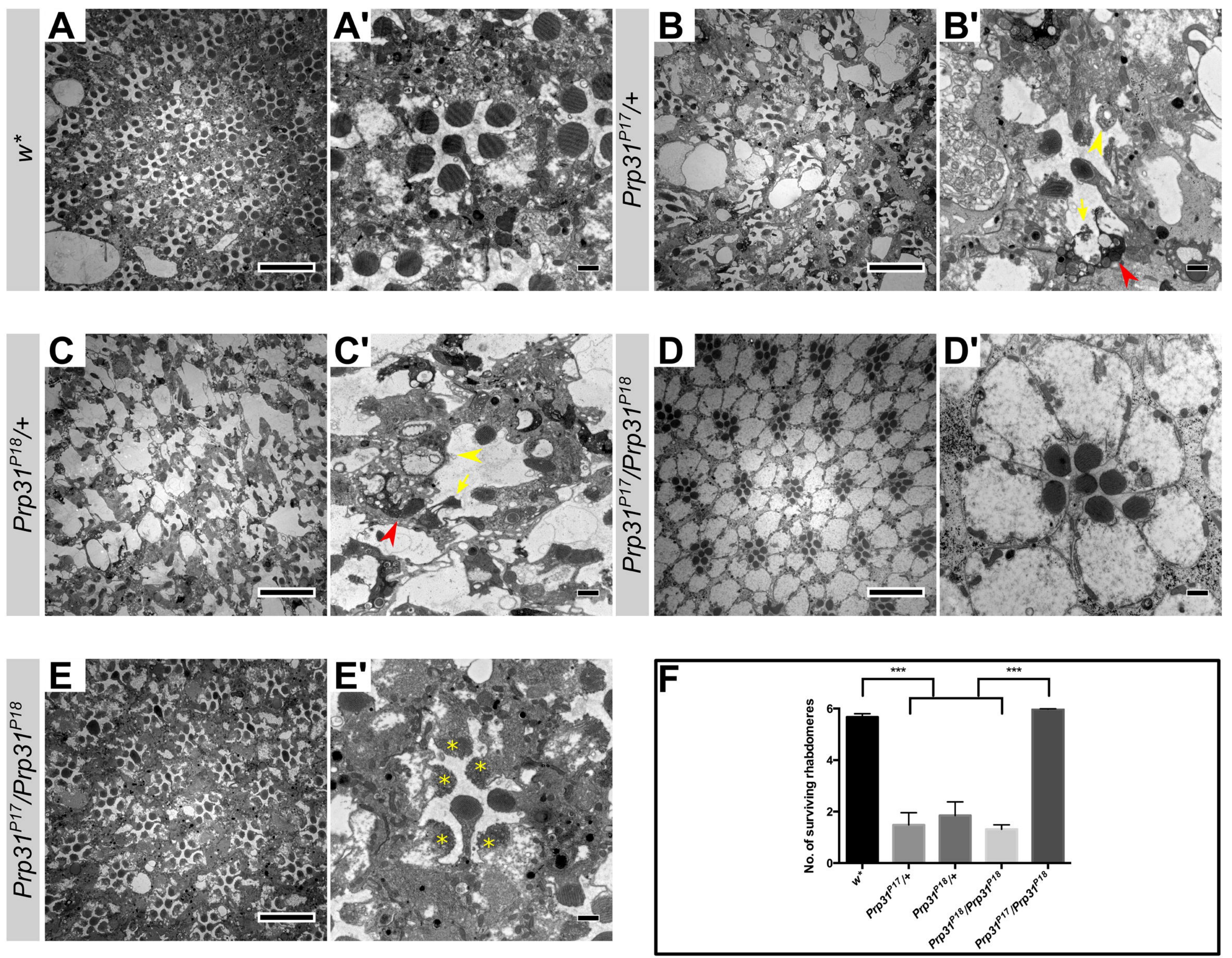

Figure 2 
A
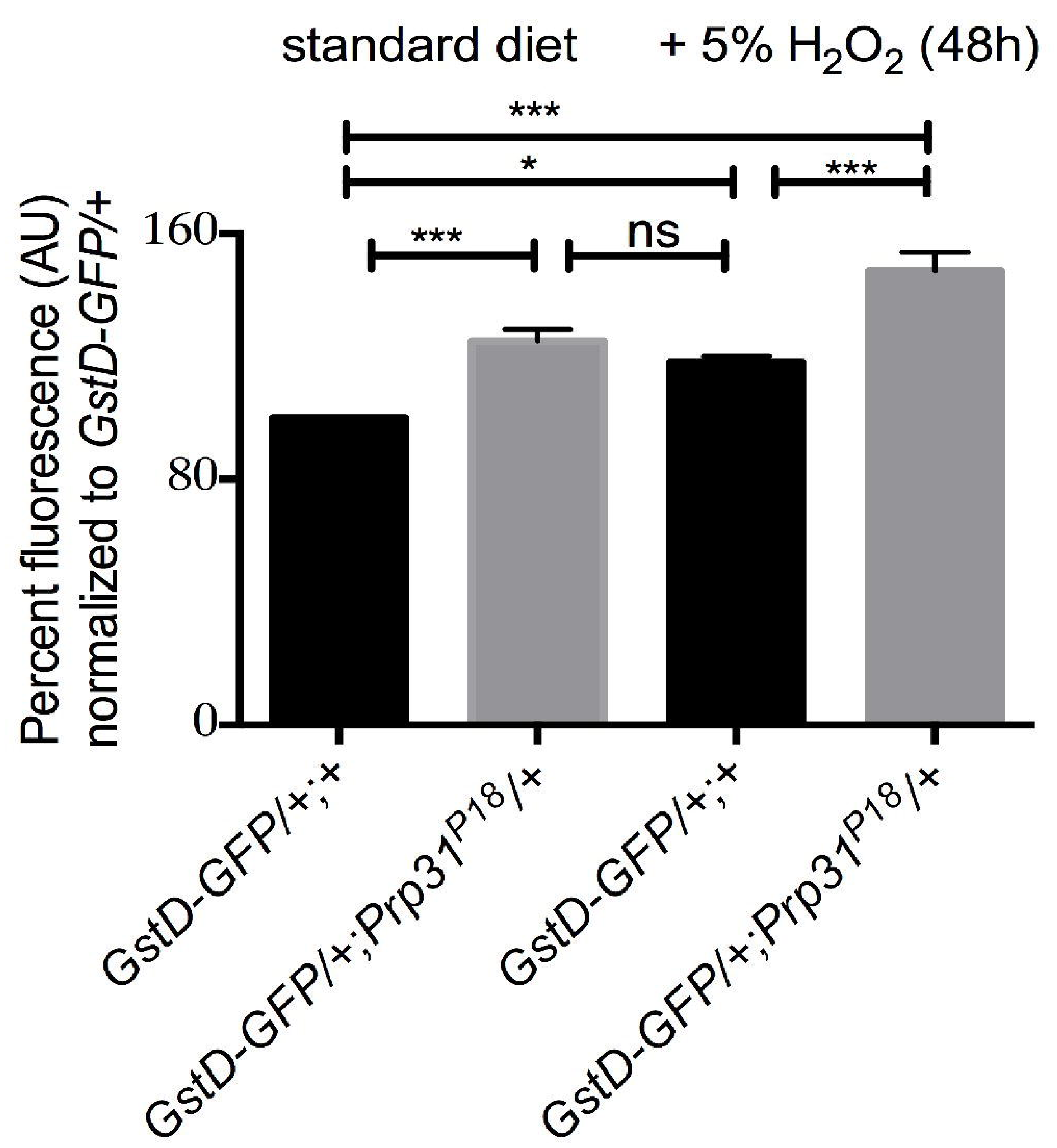

D

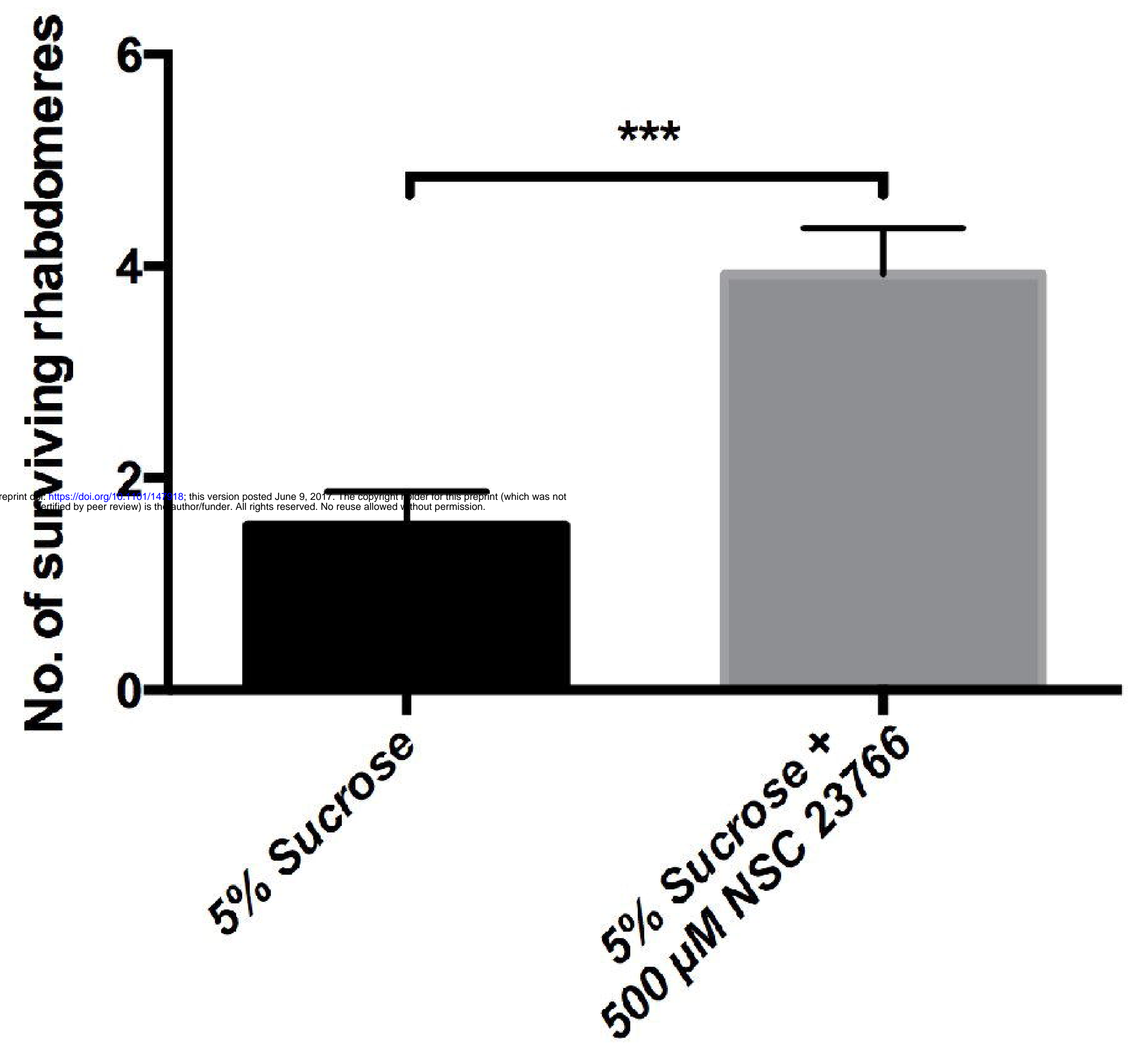

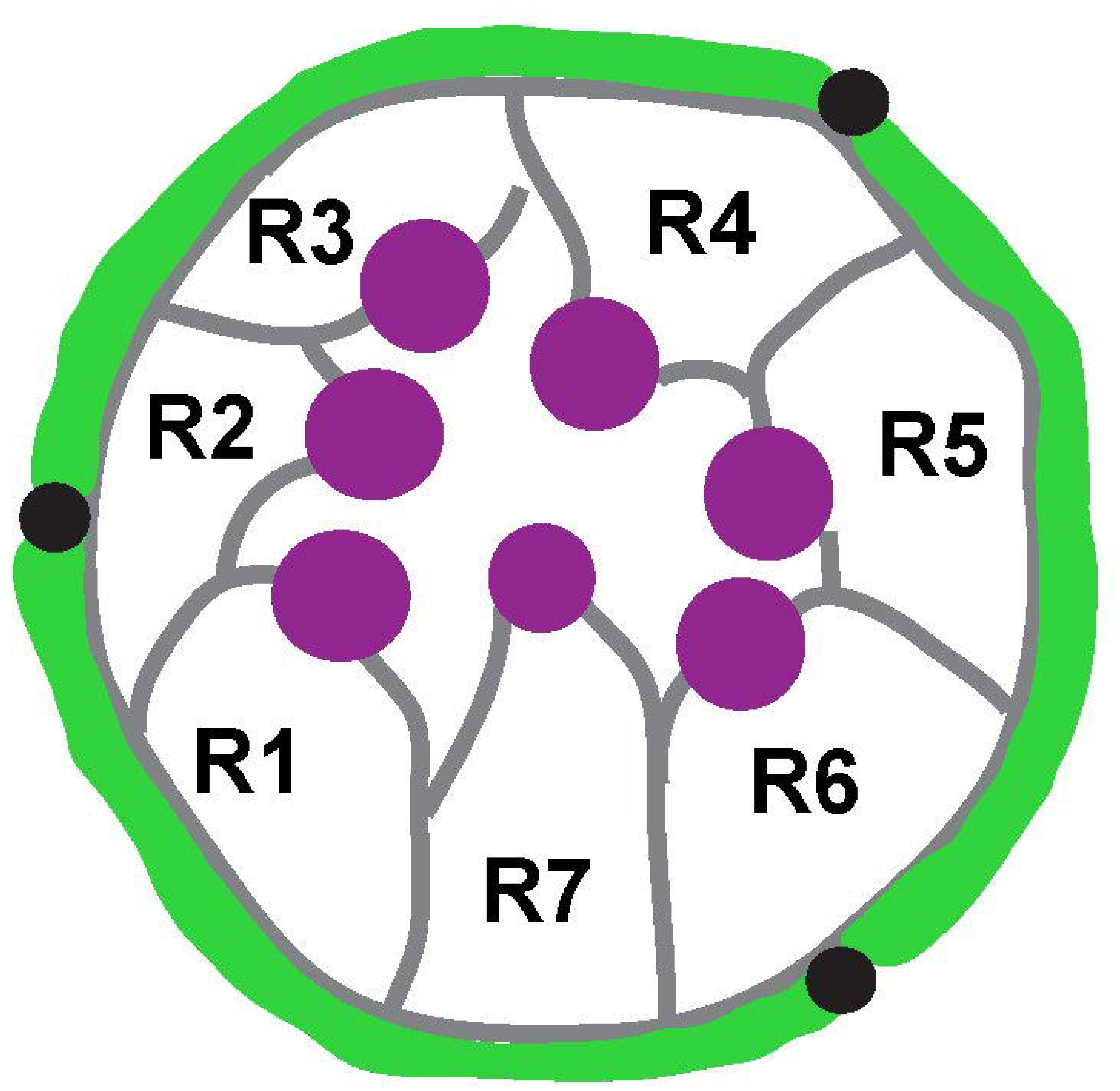

Anti-GFP/Phalloidin/Anti-Na+-K+ATPase

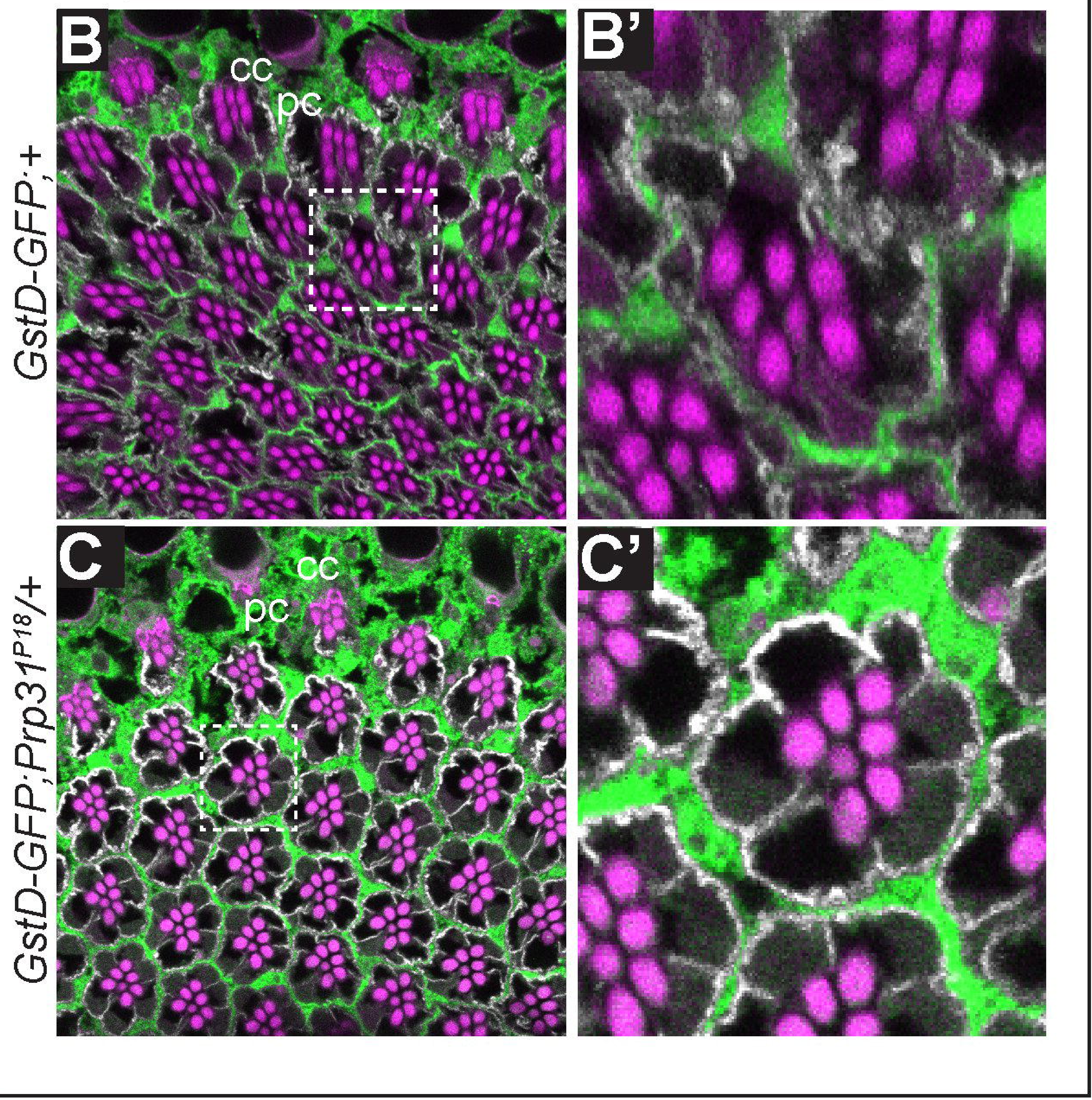

Figure 3 






